\title{
Social Presence Approach Within the Question and Answering eLearning Model:An Experiment with a Multi-Agent System
}

Cecilia Avila* | Jorge Bacca* $\mid$ Josep Lluis de la Rosa* $\mid$ Silvia Baldiris* $\mid$ Ramon Fabregat*

Recibido:

Abril 25 de 2012

Aceptado:

Mayo 30 de 2012
Institut D'informàtica i Aplicacions, Universitat de Girona, Girona, Spain cecilia.avila@eia.udg.edu, jorge.bacca@eia.udg.edu, peplluis@eia.udg.edu, baldiris@eia.udg.edu, ramon.fabregat@udg.edu

\section{Abstract}

The model of Questions Answering (QEA) for eLearning is based on collaborative learning through questions that are posed by students and their answers to that questions which are given by peers, in contrast with the classical model in which students ask questions to the teacher only. In this proposal we extend the QEA model including the social presence concept and a quantitative measure of it is proposed; besides it is considered the evolution of the resulting QEA social network after the inclusion of the social presence and taking into account the feedback on questions posed by students and answered by peers. The social network behaviorwas simulated using a Multi-Agent System to compare the proposed social presence model with the classical and the QEAA models.

Keywords: Social presence, social network, eLearning, QEAA Model, MultiAgent System.

\section{Introduction}

ocial networking creates online communities where people share interests and activities. While social networking sites like

$\checkmark$ Facebook and Twitter were not developed as educational tools they have been eagerly adopted by some educational institutions seeking new levels of student engagement and interactivity [1]. Eberhardt [2] notes that Facebook is a feature of contemporary student life and transition to university can be eased through interactions with numerous online communities. Contrary to the negative perceptions of using this very popular technology in an educational context, De Villiers [3] found that the use of Facebook for academic discussions with postgraduate distance learning students enhanced student's learning and insight. 
The approach here explored is to boost the impact of social question answering in education for increasing the competence level of groups of networked students doing question and answering to each other, similarly like Quora [4] but in controlled educational environments, this is named the not-alone experience in a collaborative environment, for instance the Q\&A model [5] in which students pose questions and peers or teacher give answers to those questions. When talking about online interaction in collaborative environments and social networks we should consider the social presence which is the degree to which a person feels 'socially present' [6].In order to analyze the behavior of the social network within the Q\&A model, we performed a set of experiments based on a Multi-Agent System that allow us to see what happen when the social network is static (in the Q\&A model do not discover new friends), or what happen when the social presence of each student is considered and if it affects the evolution of the social network.

This document is organized as follows: Section 2 describes the background and introduces the social presence measure, in section 3 a model for the social presence is proposed and its components are described and section 4 describes the simulation stages in order to check the model; finally the results of the experiments and future work are presented.

\section{A Quantitative}

28

\section{Measure of Social}

\section{Presence}

To include the social presence concept in the Q\&A model, it is proposed a quantitative measure. Based on Chen, et al. [7], the source of social presence could be classified in: "individual characteristics", "social relationships", "user interface" and "real time interactivity". According to the characteristics mentioned, the social presence features that we considered to be includedwithin the Q\&A model are:

- Number of Friends in the social network (NFriends)

- Time between proposing a question and getting Answers (TAnswer)

- Satisfaction measure with user Interface (SInterface)

- Average Time online (ATimeonline)

The social presence wasincluded in the Q\&A Model as a vector for every student as follows:

$S P v=\{$ NFriends,TAnswer, SInterface, ATimeonline $\}$

In Equation 1, the SPvis the Social Presence vector.SPv for each student that will be compared with an ideal or desirable Social Presence vector according to the cosine distance [8] and this measure represents a value that allows to identifying if a student perceives a high or a low level of Social Presence.

Socialpresence $=\operatorname{COS} \theta=\frac{S P v_{i} \bullet S P v_{j}}{\left\|S P v_{i}\right\| *\left\|S P v_{j}\right\|}$

In Equation 2, SPvi is the ideal social presence vector (target vector), and SPvj is the social presence vector of each student.

As a result in Equation 2, Social Presence shows a quantitative measure of the social presence for every student (or agent) in comparison with the target vector. This measure is a value between 0 and 1 , where values near to 0 indicates a low Social Presence and values near to 1 indicates a high level of Social Presence.

\section{Social Presence Within the QEBA Model}

\subsection{General description}

In Figure 1, the information handling of the process is depicted. There are three lay- 
ers: data, model and output. In the data layer there are two inputs: the first one is the Social Presence Level (which is measured as explained in section 2 with the cosine distance) and the second one is a set of Questions and Answers. The Social Presence Level feeds the Social Presence Model (SP Model) and the Questions and Answers feed the Questions and Answers Model (Q\&A Model) located at the model layer. As a result of applying the SP Model there is a Social Network which represents the output of the model and it is involved in the Q\&A Model in order to generate the final score for the Gained Competence Level on the output layer.

Fig. 1. Social Presence Model and Query and Answer Model

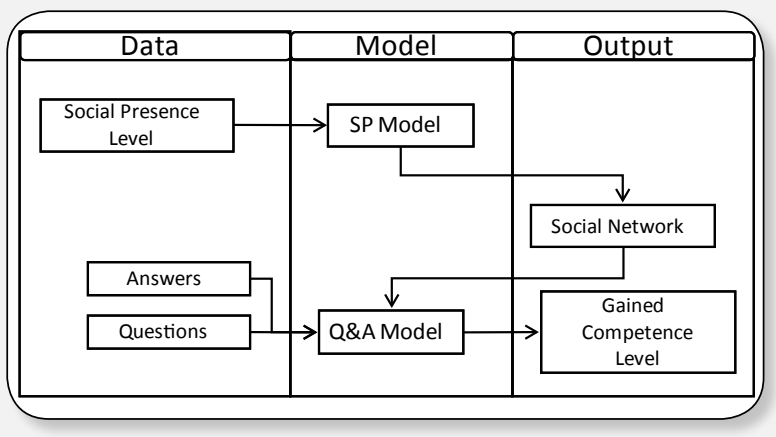

\subsection{Description of each component in the model}

- Social Presence Level. This level is calculated for every student by means of the cosine distance taking into account the issues described in section 2.

- Questions and Answers. The list of questions posed by students and the answers given by peers within a period of time, in this case 12 weeks.

- SP Model. The Social Presence Model takes the social presence level as an input and impact the Social Network evolution; at the same time affects the Q\&AModel. In this context, in the work developed by Rodrigues,Sabino and Zhou [9] there is evidence on how the interaction and contacts in social networks enrich the work of the student in the learning process:
"Throughout the learning process, the student will be confronted with questions and doubts that need clarification. The easy access to online social networks will allow all people to contact with several people. While different people do not work in the same problems they share common interests and backgrounds, thereby facilitating the sharing of information with one or both parties".

- Social Network. The Social Network has an initial contact list of $n$ friends and evolves through 12 weeks in which questions could be:

- Answered by friends of a student: In this case, a friend could explain the answersin betterempathetic ways than a teacher and with additional efforts to make other students understand [10]. In our case there is an additional bonus in the Gained Competence Level for the students who answer.

- Answered by peer students in the class (they are not in the contact list yet): In this case, if the person who answers the question is not in the contact list of the person who ask the question, and there is a friendship request previously sent, the person who ask the question accepts the friendship request and they become new friends in the Social Network and the contact list of each one is updated.

According to the friendship linksamong peers, theQuestions and Answers posted in the system impact the Social Network evolution as well as they are impacted by the behavior of students in the Social Network. In this sense the research developed by Palfreyman[11] remarks the importance of the learning behavior when people ask colleagues for information and cites the research of Garton[12] in which is described "how specific relations between an individual and his/her acquaintances may vary in their content (e.g. their topic and focus), their direction (e.g. helping or being helped) and their strength (in terms of frequency of contact, or of quantity/ 
quality of content). Such relations may accumulate over time to form strong or weak ties between individuals, which may be more or less, multiplex“. Additionally, cites the research of Borgatti and Cross [13] in which "show how people ask colleagues for information based on (a) their knowledge of the other person's expertise, (b) how positively they view the other person's skills, and (c) ease of access to the other person...".

- Q\&A Model. This model takes into account question answering among students, so students pose questions that peers answer in 12 weeksin contrast with a classical model where students ask questions only to teachers and have limited learning interaction among them [5].

- Gained Competence Level. This is the competence level which varies from 0 to 6 , according to the Q\&A Model. Competence is gained when students answer and pose questions.

\subsection{Model Behaviors}

The SP Model and the Q\&A Model are integrated as a unique model. To achieve this, there are some behaviors that managethe answering process:

- Send friendship request and the Social Presence Level. Social Presence Level impacts the Q\&AModel by means of the following condition: If there is a friendship request of student $\mathrm{A} 2$ to student $\mathrm{A} 1$ and if Social Presence Level of student A1 is larger than social presence level of student A2, we assume A1 will accept the friendship request of A2. In this sense, Social Presence takes importance due to the interaction in terms of the visibility of students. In relation to this, in [9] is proposed that: "The increased visibility of student work is very relevant through the use of online social networks. This increased visibility will improve and strengthen social relationships with other contacts" [9].
- Answer questions and friendship links. If student A1 answer the question of student A2, and they are friends in the Social Network, then student A1 receives a bonus point of 0.05 to their Gained Competence Level, otherwise, if student A2 has sent a friendship request to student A1 (which means that in this moment they are not friends), student $\mathrm{A} 1$ receives only a bonus point of 0.025 . Finally if $\mathrm{A} 1$ and $\mathrm{A} 2$ are not friends and there are not friendship request, no one receives any bonus point. We decided to assign a bonus point in the competence level taking into account the theoretical framework proposed by [10], in which the authors state that a friend or colleague could give you useful information about any topic than the information that you can find in a web site.

- Evaluate other causes. Any other cause which is raised from the interaction of the students in the social network through question posing and answering that impact the Social Presence Model.

The simulation of these behaviors is performed by a system with a Multi-Agent System, which is described in section 4 .

\section{Simulation Stages and Intelligent Agents}

Section 4.1 is a brief presentation of the developed system and section 4.2 presents how the initialization of the social network is performed. Also we considered 2 simulation stages. The first one is an experiment in which a Social Network is initialized witha group of 8 students (represented by intelligent agents) and it is described in section 4.3. The second one considers an experiment in which the Social Network evolves and it is described in section 4.4 .

\subsection{Intelligent Agents}

In order to perform the experiments, the 
system was developed with a group of 8 agents. As shown in Figure 2, the MultiAgent System interacts with the Q\&A Model by means of four types of agents: student, peer, friend and requested friend. Competence Level, Social Presence and Contact List are the main features for the student's agent. The initial Competence Level will be updated through the simulation period (12 weeks), the Social Presence is calculated with the proposed quantitative measure and the Contact List is updated according to the interaction in the Social Network. All of them are modified according to the behaviors of each agent within the interaction with others.

On the other hand, the Social Network is defined during the process of posing questions and answering, and each student's agent has behaviors to achieve this (send friendship request, pose and questions). As is considered by Neji and Ammar [14], agents can be designed to imitate human behavior, in this work agents imitate the behavior of a group of students interacting in a social network.

\subsection{Initializing the Social Network}

Following the same example of Ermalai and de la Rosa [5] there is a stage with 8 students (which are represented by intelligent agents in the experiment), and the Social Network is organized by assigning an initial number of friends for every agent. As a result of initializing theSocial Network we have two types of relationships. The first one is a bidirectional type, for example whenstudent A5 sends a friendship request to another student and this student accepts that request.

The second one is a unidirectional type, for example when student $\mathrm{A} 5$ sends a request to student $\mathrm{A} 7$ and this request is not answered. In this context, the Social Network works in the same way than Facebook, when people send friendship request to other person, he/ she have to accept the request in order to keep in touch with the other person [15].

Once the initial Social Network has been created, with a group of friends for every student, and a set of friendship request have been sent to others in the Social Network, the process of posing questions and answers is started. According to[9]when people interact and share information, this process "might encourage people to learn together, sharing

Fig. 2.Multi-Agent System Architecture in the model

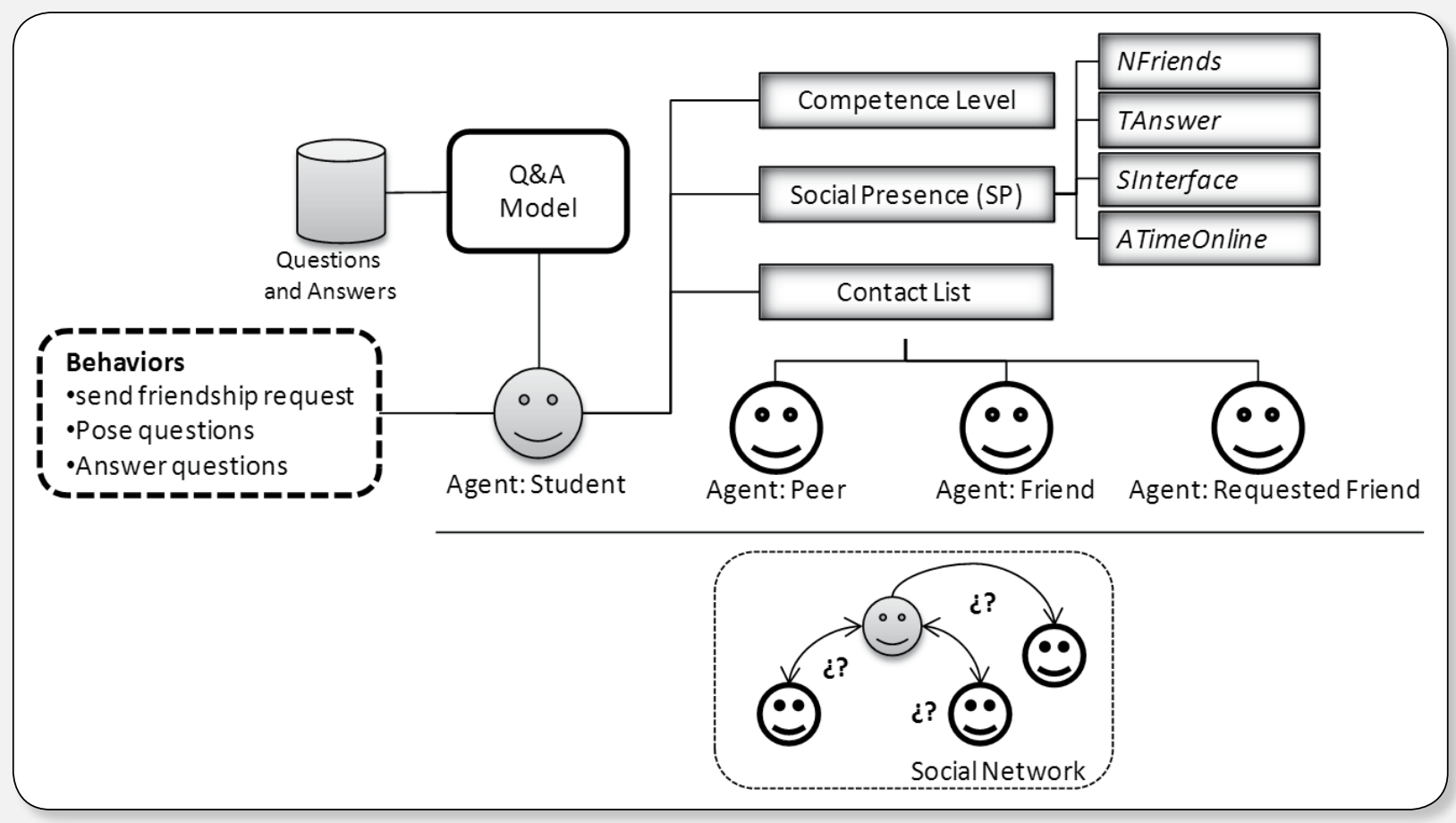




\section{Respuestas}

Año 17

No. 1

Junio 2012

ISSN 0122-820X views and content relating to the matters in question".

\subsection{Experiments with a static social network}

In this stage, after the Social Network initialization, students pose questions and peers answer those questions within 12 weeks in which no new links in the Social Network are created; so at the end of the simulation the Social Network structure does no change. In this stage the following conditions are taken into account:

- When a student answers a question posed by a friend receives an additional bonus point as described in section 3.3 which is added to the Gained Competence Level.

- When a student answers a question posed by a peer (not a friend), the social relationship with the peer does not change. In this case the interaction by means of question posing and answering do not create new friendship links.

Figure 3 shows the results of an experiment with a static social network. The $y$-axis in the figure represents the Gained Competence Level; the $\mathrm{x}$-axis is the time in days (in 12 weeks) and each line in the stair stepgraph shows the evolution of each student. In this case we are not considering the Social Presence Level yet; it is only a demonstration of the behavior in the Social Network and the Gained Competence Level of students.

Fig. 3. Simulation stage of the static social network

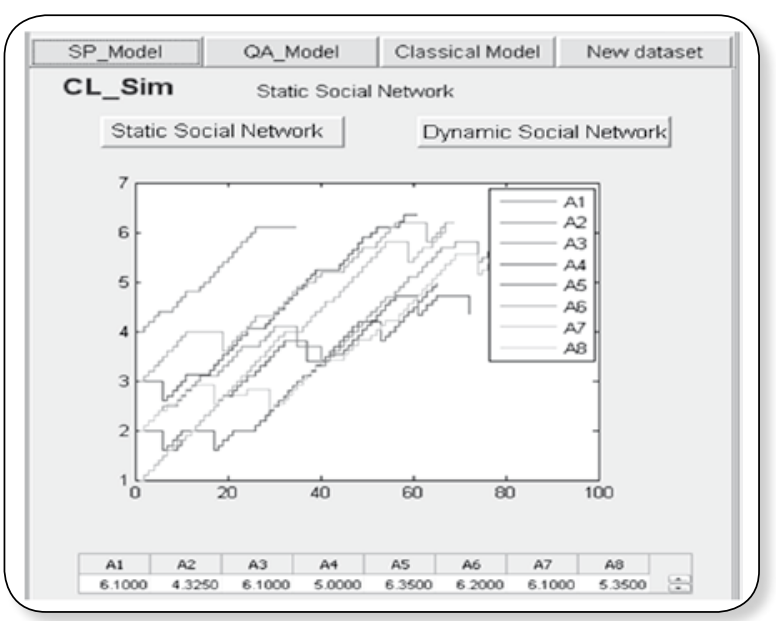

\subsection{Experiments with a dynamic Social Network}

In this stage, once the social network has been initialized, students pose questions and peers answer the questions within 12 weeks in which new links could be created due to friendship requests sent to other peers which are accepted during the process when interaction occurs in the Social Network (questions posed are answered by peers). The following conditions are taken into account:

- If student $\mathrm{A} 1$ has sent a friendship request to student A2, student A2 has proposed a question and student A1 answers the question, student $\mathrm{A} 1$ receives a bonus point of 0,025 to the Gained Competence Level and accept the friendship request, so there is a new link of friendship in the Social Network.

- If student A1 and student A2 are friends and student A1 answers a question proposed by student $A 2$, student $A 1$ receives a bonus point of 0,05 to the Gained Competence Level.

- If student A1 and student A2 are not friends and there is not friendship requests sent and student $\mathrm{A} 1$ answers a question proposed by student $\mathrm{A} 2$, then bonus point are not given to anybody.

Fig. 4. Experimentwith dynamic social network

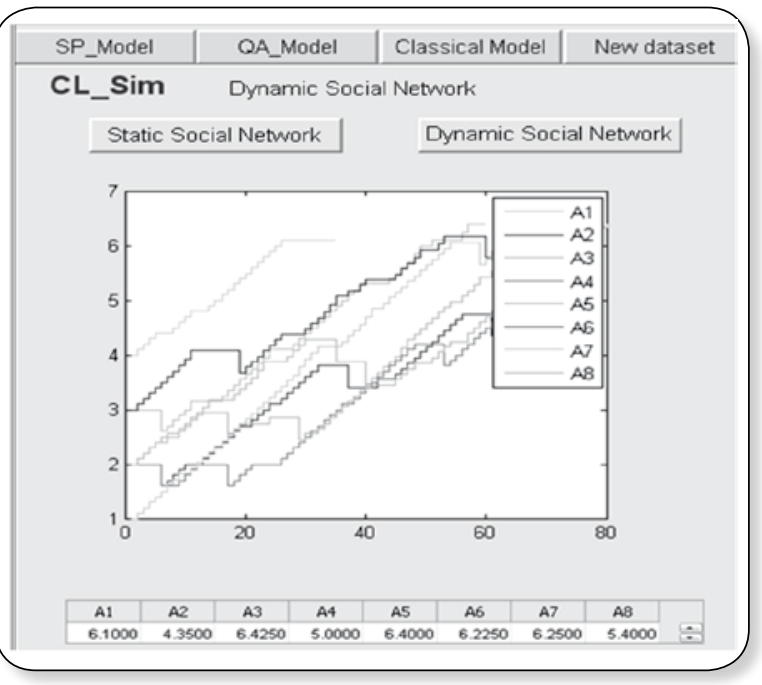


As a result at the end of the experiment the Social Network structure has been updated than the initial structure which means that students have new friends that comes from the interaction in questions and answers and taking into account the social presence level of each student. Figure4 shows the results of anexperiment with dynamic social network.

\section{Discussion and Results}

The following tables show the results obtained in the experiments described in sections 4.2 and 4.3 , and allow comparing the gained competence for every student in the end of the experiment. Table 1 shows the results obtained at the end of the experiment stage with a static social network and table 2 shows the results obtained at the end of the experiment stage with a dynamic social network.

According to the results shown in table 1 and table 2, we observe that the results of the dynamic network are better than the results obtained in the static network. It is important to take into account that a same dataset was used for both experiments. From the results we can observe that students reach higher values of competence with a dynamic network which social relationships were raised due to the interaction by means of question posing and answering. For instance, students A2, A3, A5, A6, A7, and A8 obtained better results than in classical or Q\&A Model. It means that in a dynamic social network in the context of a learning environment where students interact by means of question posing and answering, students obtain better results in the Competence Level.

Table 1. Results of the simulation with the static network

\begin{tabular}{|c|c|c|c|c|c|c|c|c|}
\hline Agents / Students & A1 & A2 & A3 & A4 & A5 & A6 & A7 & A8 \\
\hline Gained Competence Level & 6.1 & 4.325 & 6.1 & 5.0 & 6.35 & 6.2 & 6.1 & 5.35 \\
\hline
\end{tabular}

Table 2. Results of the simulation with the dynamic network
On the other hand in table 3 the results obtained for the gained competence (after 10 simulations) are summarized as well as the general average in each model. As is shown in the general average column, the dynamic social network and SP Model has the better result in comparison with the others concluding that the social presence level and the evolution in an social network (we defined this feature as dynamic social network) are factors that influence in a positive way the eLearning process when there is an interaction through the question posing and answering strategy.

Table 3. Comparison of the average in gained competence level

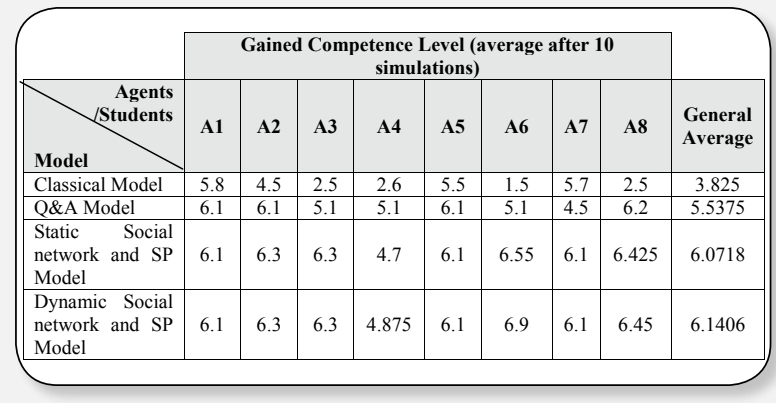

Table 4 shows a comparison of the average percentage improvement in the gained competence from each model. The results are: an improvement of $60.53 \%$ in the Dynamic SP Model (or Dynamic Social network and SP Model) when it was compared with the Classical Model; a 10.89\% in the Dynamic SP Model when it was compared with the Q\&A Model and a 1.13\% in the Dynamic SP Model when it was compared with Static Social network and SP Model.

Table 4. Average percentage improvement in the gained competence from each model 


\section{Conclusions and Future work}

The quantitative measure of the Social Presence Level in each student allows updating the Social Network, taking into account the interaction of students in the network by means of question posing and answering from the Q\&A Model.

The quantitative measure of the social presence in the system could be used in order to recommend activities for students that have a lower social presence level.

Social Network and Social Presence has been integrated with the Q\&A Model in order to introduce social factors in the collaborative learning process.

On the other hand by means of the experiments we can observe how the MultiAgent System evolves by interacting in a social network through question posing and answering. As a result of the experiments we observed a better performance in the dynamic social network (in which is included the social presence concept) than the other compared models, in terms of the gained competence obtained by students.

\section{References}

1. Boon, S. and Sinclair, C.: A World I Don't Inhabit: Disquiet and Identity in Second Life and Facebook. Educational Media International (46:2), 99 - 110 (2009).

2. Eberhardt, D. M.: Facing up to facebook. About Campus, 12 (4), 18-26 (2007)

3. De Villiers, R.: Academic Use of a Group on Facebook, in Proceedings of InSITE Confer-ence,Informing Science Press, Bari and Cassino, Italy, pp. 173-190. (2010)

4. Quora, http://www.quora.com/

5. Ermalai,Iasmina and De la Rosa, Josep.:Analysis of the benefits of collective learning through question answering. In: 4th International CSEDU 2012. Portugal (2012)

6. Leh, A. S.: Computer-Mediated Communication and Social Presence in a Distance Learning Environment. International J. of Educat. Telecommunic., 7(2). 109-128 (2001)

7. Chen, N., Kinshuk, Wei, C., Wang, M.: A Framework for Social Presence in Synchronous Cyber Classrooms. In:Ninth IEEE International Conference on Advanced Learning Tech-nologies 2009, pp.40-44, 15-17. IEEE Press, New York (2009)

8. Manning, C. D., Raghavan, P., Schutze.: Introduction to information Retrieval. Cambridge University Press. pp. 121 (2008)

9. Rodrigues, J., Sabino, F., Zhou, L.: Enhancing e-learning experience with online social networks. IET Commun., 5 (8),1147-1154 (2011)

10. Connole, G., Culver, J.: The design of Cloudworks: Applying social networking practice to foster the exchange of learning and teaching ideas and designs. Computers \& Education 54. 679-692 (2010)

11. Palfreyman, D.: Social context and resources for language learning.System 34(3) 352-370 (2006)

12. Garton, L., Haythornthwaite, C., Wellman, B.: Studying online social networks. Journal of Computer Mediated Communication 3 (1). (1997)

13. Borgatti, S., Cross, R.: A relational view of information seeking and learning in social networks. Management Science 49, 432-445. (2003)

14. Neji $M$ and Ben Ammar M.: Agentbased Collaborative Affective e-Learning Framework. The ElectronicJournal of e-Learning., 5(2), 123-134. (2007)

15. Traud, Amanda, Mucha, P., Porter, M. "Social structure of Facebook networks". In press. Physica A - Statistical Mechanics and its Applications. (2011) 